\title{
Radical-Mediated Synthesis of Sesquiterpene Lactones
}

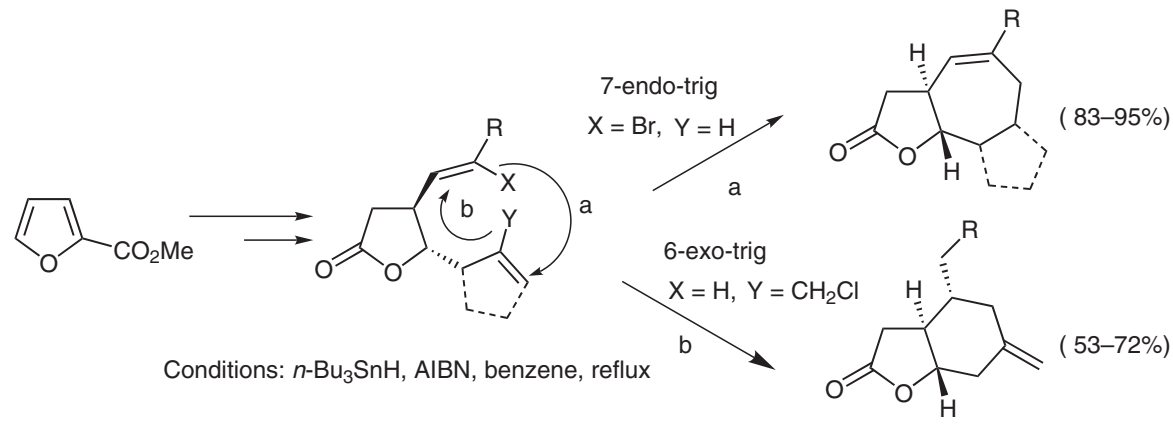

Significance: Starting from inexpensive methyl 2furoate, via the intermediate cyclopentenylbutenolide (4 steps, 26\% overall yield), bi- and tricyclic $\gamma$-butyrolactone cores can be formed stereoselectively and in good yields via Y-substituent - directed radical cyclization. Using $\mathrm{R}=\mathrm{Br}, \mathrm{CN}, \mathrm{CO}_{2} \mathrm{Et}$, COMe substitution, reactions proceed in acceptable yields.
Comment: trans-Fused seven- or six-membered rings are widespread frameworks in a variety of natural products such as guaianolides. This stereoselective strategy may provide new useful synthetic approaches to such derivatives and other natural products containing similar ring systems which to date have been rather limited. 